\title{
Interplay between Structure and Electronic Properties in Organic Conductors
}

\author{
Hidetoshi Fukuyama ${ }^{1}$, Hitoshi Seo, Hiori Kino \\ Institute for Solid State Physics, University of Tokyo, Minato-ku, Tokyo 106-8666, Japan
}

\begin{abstract}
It is known that the ground states of organic conductors have a diversity reflecting the spatial arrangement of the constituent molecules within the unit cell. A systematic theoretical search for the unifying view behind such possible ground states has been made based on the Hartree-Fock mean field approximation not only to the onsite but also to intersite Coulomb interactions with special emphasis on the families of typical organic conductors $(\mathrm{TMTCF})_{2} X$ and $(\mathrm{ET})_{2} X$.
\end{abstract}

Keywords: organic conductor; Hartree-Fock approximation; Mott insulator; charge ordering

\section{Introduction}

The insulating states of solids are realized either by the absence or the localization of carriers; the former is the band insulator and the latter cases are due to either disorder (Anderson localization) or the mutual Coulomb interactions. In the particular case of the non-degenerate half-filled band the on-site Coulomb interaction leads to the Mott insulating state, while in the case of the quarter-filled bands the mutual Coulomb interaction of not only on-site but also between different sites play important roles leading to the state where electrons are localized every other sites as in the Wigner crystal. This is a kind of the charge ordering $(\mathrm{CO})$ phenomena. In both cases of Mott insulators and Wigner

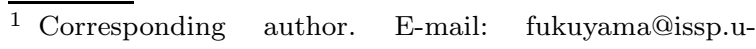
tokyo.ac.jp

Preprint submitted to Physica $B$ crystals the electron spins are active and then the transport and the magnetic properties are closely interrelated.

In recent years there are much of the research activity in organic conductors which quite often exhibit metal-insulator transition as a function of temperature, pressure or magnetic field. The experimental studies have elucidated the existence of a diversity in interesting electronic properties even if the constituent molecules and their composition ratio are the same. Typical examples are $(\mathrm{TMTCF})_{2} X(\mathrm{TMTCF}=\mathrm{TMTTF}, \mathrm{TMTSF})$ and $(\mathrm{ET})_{2} X(\mathrm{ET}=\mathrm{BEDT}-\mathrm{TTF})$. In these examples, $X$ represent anions attracting almost one electron, i.e. $X^{-}$, and then the conduction processes are due to the $\pi$-bands of the donors, TMTCF ${ }^{1 / 2+}$ or $\mathrm{ET}^{1 / 2+}$ which is quarter-filled if all these molecules are equivalent. However, in some cases such as quasi-one-dimensional (TMTCF $)_{2} X$ with 
two donor molecules in a unit cell along the chain direction, there exists a small degree of the dimerization, which results in neither idealistic quarterfilled nor half-filled bands. On the other hand in cases of $(\mathrm{ET})_{2} X$ there often are four ET molecules in a unit cell with very anisotropic transfer integrals between molecules, which fact is the cause of the diversity in electronic properties of these systems, e.g. ground states with antiferromagnetism (AF), superconductivity (SC), Peierls and spinPeierls (SP) distortions, spin gap (SG) and so on.

In this paper we will briefly review our recent theoretical studies based on the Hartree-Fock (HF) approximation, searching for the simple unifying view behind these apparent complexity. The effects of quantum fluctuations, which are not taken into account in this approximation, are then assessed based on the results thus obtained.

\section{HF approximation for complex unit cell}

When the electronic state at each site is properly represented by one molecular orbital, the Hamiltonian will be expressed as follows,

$$
\begin{aligned}
H=\sum_{<i, j>} & \sum_{\sigma}\left(-t_{i, j} a_{i \sigma}^{\dagger} a_{j \sigma}+\text { h.c. }\right) \\
& +\sum_{i} U n_{i \uparrow} n_{i \downarrow}+\sum_{<i, j>} V_{i, j} n_{i} n_{j}
\end{aligned}
$$

Here $\mathrm{t}_{i, j}$ is the transfer integrals between molecules $i$ and $j$, which can be calculated within the extended Hückel approximation, and $U$ and $V_{i, j}$ are on-site and intersite Coulomb interactions, respectively, and $n_{i s}=a_{i s}^{\dagger} a_{i s}$ and $n_{i}=n_{i \uparrow}+n_{i \downarrow}$. In the actual treatment of the Coulomb interactions the $U$ and $V_{i, j}$ terms are approximated within the $\mathrm{HF}$ and Hartree approximation, respectively[1] (we use the term HF below for simplicity). This HF approximation is a durable and systematic procedure for the search of possible ground states. It should be kept in mind, however, that the quantitative aspects of results of this approximation should not be taken literally but that the results will be a basis for the further detailed theoretical studies.

\section{HF calculations}

\section{1. $(\mathrm{TMTCF})_{2} \mathrm{X}$}

This family has one-dimensional structures with the alternation in the transfer integrals along the chain direction. This dimerization result in a halffilled band, instead of the quarter-filling which is expected if all TMTCFs are equivalent. However it is to be noted that this dimerization is very weak compared with the mutual Coulomb interactions as will be easily inferred by the smallness of the dimerization gap in the band structure, and then half-fillingness of this system should be taken with care.

The experimental phase diagram of this family on the plane of pressure and temperature, is proposed by Jérome[2]. As the pressure (external as well as chemical) increases, the ground state of this system varies as $\mathrm{SP}$, commensurate $\mathrm{AF}$ (C-AF), incommensurate spin-density-wave (IC-SDW), SC and paramagnetic metallic (PM) states. An important feature in this phase diagram is the existence of temperature $\mathrm{T}_{\rho}$, where the resistivity shows minimum, in the systems whose ground states are $\mathrm{SP}$ and $\mathrm{C}-\mathrm{AF}$. In this low pressure regime, the localization of the charge sets in at $\mathrm{T}_{\rho}$ as the temperature is decreased, independently of the magnetic ordering. HF calculations[3] for the ground states in these cases indicate that the spatial pattern of the ordered spins in the C-AF phase is rather close to that of $\mathrm{CO}$ state (as in Wigner crystals) where the spins with $\mathrm{S}=1 / 2$ in every other site, rather than a Mott insulator spin $\mathrm{S}=1 / 2$ on each dimers which is the case for strong dimerization. The antiferromagnetic pattern in this case, which is schematically described as $(\uparrow 0 \downarrow 0)$ has actually been observed in some TMTTF compounds[4]. The dielectric permitivity measurement[5] on (TMTTF $)_{2}$ Br also suggest the 
$\mathrm{CO}$ state. Moreover, the tencency to $\mathrm{CO}$ has been observed directly by NMR in different but similar systems (DI-DCNQI) ${ }_{2} \mathrm{Ag}[6]$. These results indicate that the origin of the insulating state in the region of low pressure will be a CO state driven by the intersite Coulomb interaction, $V$, rather than the simple Mott insulators.

\section{2. $\kappa-(E T)_{2} X$}

The family of $(\mathrm{ET})_{2} X$ has many polytypes with a variety of ground state, of which the crystal structures are basically the same but spatial arrangements of ET molecules in a unit cell are different, which result in drastically different ground states as described below.

$\kappa-(\mathrm{ET})_{2} X$, which has 4 ETs in a unit cell, has the structure shown in Fig. 1 (a) where the transfer integrals between molecules are also indicated. This family exhibits a phase diagram with an interesting feature of having boundaries between AFI and $\mathrm{SC}[7]$, whose symmetry can be different from that of BCS[7]. As seen in Fig. 1 (a) the transfer integral $t_{b 1}$ is relatively larger than any others implying the formations of the dimers between two molecules connected by this $t_{b 1}$. Actually, the band structures in the paramagnetic state is such that there exists a clear band-gap between the higher two and lower two bands, which are due to the antibonding and bonding orbitals within the dimers, respectively. The HF calculations reveal that in the presence of large $t_{b 1}$, which is the crucial parameter characterizing the degree of dimerization, and of large $U$ an $\mathrm{AF}$ insulating (AFI) state shown in Fig. 2 (a) emerges[8]. This AFI state is same as the $\mathrm{AF}$ of spin $\mathrm{S}=1 / 2$ on each dimer[9]. Once the effective value of $U$, i.e. $U / W$, is reduced by pressure, $\mathrm{HF}$ calculations predicts the transition from AFI to PM, which can be SC as in the above case of (TMTCF $)_{2} X$. (a)

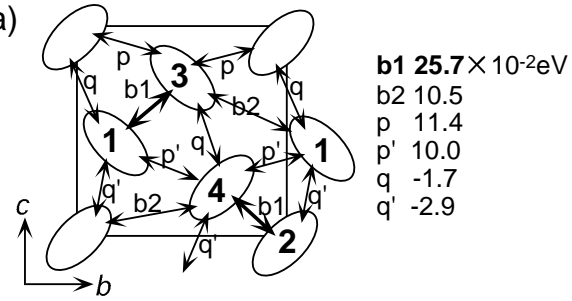

(b)

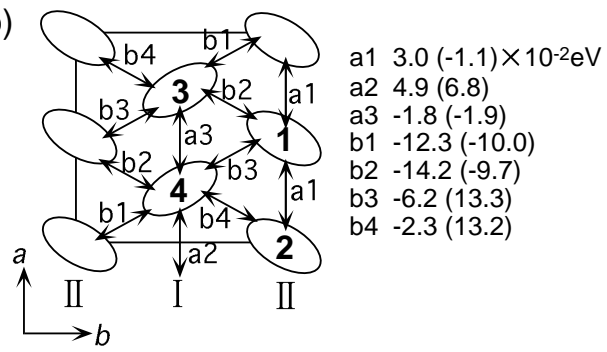

(c)

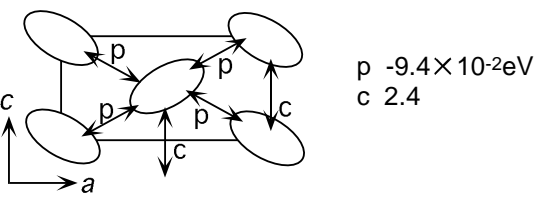

(d)

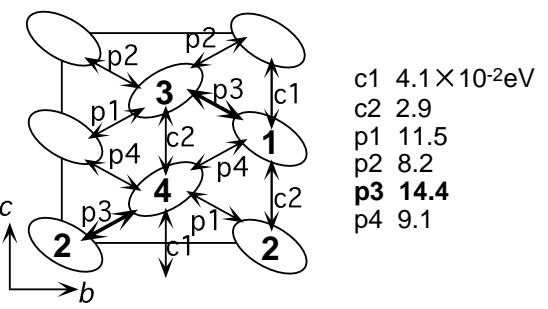

Fig. 1. Schematic representations of the structures in the donor plane for $(\mathrm{ET})_{2} X$ compounds; $\kappa$-type (a), $\theta$-type (b), $\theta_{d}$-type (c) (see the text for the notation of $\theta_{d}$ ) and $\alpha$-type (d). ET molecules are represented as elipses, and the intermolecular transfer integrals are also shown. (see ref. $[8,12])$

\section{3. $\alpha-(E T)_{2} I_{3}$}

The temperature dependence of resistivity of this system is known to have interesting features, i.e. at ambient pressure the resistivity sharply rises at around $130 \mathrm{~K}$ as $T$ is lowered but with apparently finite limiting value towards $T=0$, while at $p=12$ kbar the temperature dependence of the resistivity is metallic towards $T=0[10]$. In the almost insulating state at low temperature at ambient pressure, the spin susceptibility is suppressed isotropically indicating a non-magnetic 
(a)

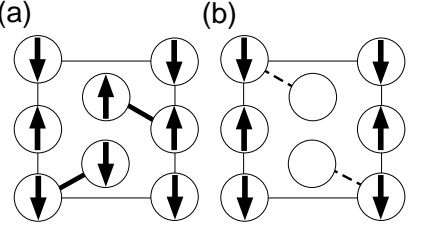

Fig. 2. A schematic reprensentation of the insulating states of $\kappa-(\mathrm{ET})_{2} X$ (a) and $\alpha-(\mathrm{ET})_{2} \mathrm{I}_{3}$ (b), deduced from $\mathrm{HF}$ calculations [8]. The thick bonds in (a) and the dotted bonds in (b) correspond to the transfer integrals $t_{b 1}$ and $t_{b 4}$, repectively.

ground state with a $\mathrm{SG}[11]$. This polytype also has 4 ETs in a unit cell, whose spatial arrangement is shown in Fig. 1 (b). For this structure, all of the four bands are weakly separated and the Fermi level is located roughly between top-most and the next-top bands. The HF calculations with only $U$ predict that in the case of small values of the transfer integral $t_{b 4}$, which turns out to be the key factor to determine the degree of band overlap between these two bands, an AFI state different from that in the $\kappa$-phase, as shown in Fig. 2 (b) is stabilized[8]. This AFI state has one-dimensional arrays of spin, whose magnitude is close to $S=1 / 2$, caused by $\mathrm{CO}$, which we call here 'stripes', between columns along the $a$-axis. However, similar calculations including intersite Coulomb interactions predict the stripes along the $b$-axis[12]. This fact indicates that the spatial pattern of $\mathrm{CO}$ in this system may be very sensitive to the physical parameters. Hence the determination realized of this $\mathrm{CO}$ is an experimental challenge.

\section{4. $\alpha-(E T)_{2} M H g(S C N)_{4}$}

The spatial arrangement of ETs in this system are similar to $\alpha$ - $(\mathrm{ET})_{2} \mathrm{I}_{3}$ though the values of transfer integrals are different as shown in the parenthesis in Fig. 1 (b), where the value of $t_{b 4}$ is quite larger than in the case of $\alpha-(\mathrm{ET})_{2} \mathrm{I}_{3}$. This system is intermediate between $\alpha-(\mathrm{ET})_{2} \mathrm{I}_{3}$ and $\kappa$ - $(\mathrm{ET})_{2} X$; if the degree of dimerization in $\kappa$-(ET) $)_{2} X$ and the degree of the band-overlap in $\alpha-(\mathrm{ET})_{2} \mathrm{I}_{3}$ are varied, this system is realized[1,8]. This affords a unified view on these three different polytypes, as seen in

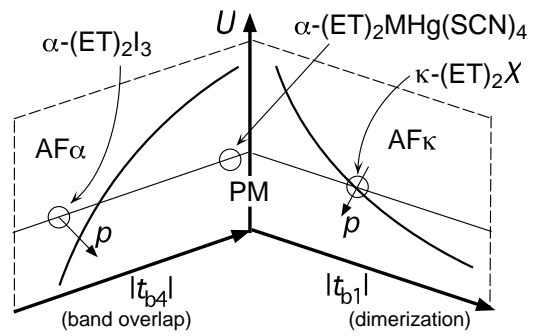

Fig. 3. A schematic phase diagram of the $(\mathrm{ET})_{2} \mathrm{X}$ compounds[1]. $\mathrm{AF}_{\kappa}$ and $\mathrm{AF}_{\alpha}$ are the $\mathrm{AF}$ states shown in Fig. 2 (a) and (b), respectively. Arrows with $p$ denotes the effects of applied pressure.

the HF phase diagram shown in Fig. 3.

\section{5. $\theta-(E T)_{2} X$}

$$
\theta-(\mathrm{ET})_{2} M M^{\prime}(\mathrm{SCN})_{4}\left(M=\mathrm{Rb}, \mathrm{Cs}, \mathrm{Tl}, M^{\prime}=\mathrm{Zn}, \mathrm{Co}\right)
$$
are well studied experimentally in recent years, and the possibility of $\mathrm{CO}$ as the origin of its insulating behavior has been proposed[13]. Depending on the anion $\left[M M^{\prime}(\mathrm{SCN})_{4}\right]^{-}$or even on the cooling rate, there are two different arrangements of ET molecules in their two-dimensional layers, as shown in Fig. 1 (c) and (d)[14], which we call here $\theta$-type and $\theta_{d}$-type, respectively. The $\theta$-type salts has a simply quarter-filled band, whereas in $\theta_{d}$-type salts the bands are split into two which are slightly seperated due to the weak dimerization: $t_{p 3}$ in Fig. 1 (d) is larger than the others. $\mathrm{HF}$ calculations on each structure suggest various stripe-type CO states are stabilized[12], where the actual charge pattern is sensitive to the values of the parameters of systems. We can infer from optical conductivity[15] data that the stripes are along $c$-axis for $\theta$-type and in the $b$-direction along the bonds with $t_{p 4}$ for $\theta_{d}$-type structures, respectively, which are among the candidates in our results of HF calculations.

\section{Magnetic properties -Effects of quantum fluctuation-}

As seen in the previous section, the HF calculations indicate the arrangements of spins when the 
charges are localized. However, the quantum fluctuations are neglected in the HF calculations, and hence these results can be different for some cases from the observed magnetic properties. Such effects of quantum fluctuations will be properly incorporated by mapping the situation to the $S=1 / 2$ Heisenberg Hamiltonians where these spins are interacting by the superexchange interactions deduced from the stuctures.

\section{1. $(\mathrm{TMTCF})_{2} X$}

Since the localized state along the chain direction in the region of low pressure are the $\mathrm{CO}$ state as noted in $\S 3.1$, the spin degree of freedom can be represented by the quasi-one-dimensional $\mathrm{AF}$ Heisenberg model with both the coupling to the lattice, $\lambda$, and interchain superexchange interaction $J_{\perp}$. If the former is dominant, the SP state is realized, but as the latter gets important, e.g. by the external pressure, it had been theoretically predicted that there will be a first order phase transition from the SP state to the AF state[16], which is consistent with the observed properties.

\section{2. $\kappa-(E T)_{2} X$}

According to the HF calculations, the AFI state of this system is as shown in Fig. 2 (a), which indicates the existence of (almost) localized spins on the triangular lattice with slightly anisotropic couplings [1,9]. The NMR measurements[7] show the existence of the AF long range order, with AF pattern identical to our results, which is consistent with the absence of SG in calculations on the case of the $\mathrm{S}=1 / 2$ isotropic triangular lattice model.

\section{3. $\alpha-(E T)_{2} I_{3}$}

The CO state with the resultant spins along the $a$-axis as shown in Fig. 2 (b) as well as those along the $b$-axis proposed in ref. [12] will both result in a one-dimensional spin system with alternating couplings. The magnetic susceptibiliy data seems to suggest the latter case, since the magnitude of the superexchange couplings may be too small for the former case if we assume that they can be described by the simple superexchange relation i.e. $J=4 t^{2} / U$.

\section{4. $\theta-(E T)_{2} X$}

Similar discussions to the case of $\alpha-(\mathrm{ET})_{2} \mathrm{I}_{3}$ can be made in this polytype since we found that a stripe type $\mathrm{CO}$ is also realized in this family. In the $\theta$-type compounds, the magnetic susceptibility shows a Curie-like behavior without any magnetic ordering down to lowest temperatures. In contrast, $\theta_{d}$-phase exhibits a magnetic susceptibility with a Bonner-Fisher behavior in the intermediate temperature range and below around $10 \mathrm{~K}$, a spin gap behavior is observed. The stripe type $\mathrm{CO}$ with patterns denoted in $\S 3.5$ are consistent with these measurements, since the values of $J=4 t^{2} / U$ with $t=$ $t_{c}$ and $t_{p 4}$ along the stripes are of the order of few $\mathrm{K}$ and $100 \mathrm{~K}$, respectively. Then the SG behavior observed in the $\theta_{d}$-phase may be due to the SP transition.

\section{5. $\lambda-(B E T S)_{2} X$}

This family with $X=\mathrm{GaX}_{z} \mathrm{Y}_{4-z}$ has 4 BETS (a sulfer analog of ET) molecules in a unit cell as in the case of $(\mathrm{ET})_{2} X$. Experiments have disclosed that the phase diagram of this system shares common features with $\kappa$-(ET) ${ }_{2} X$ but with remarkable difference, i.e. the insulating state next to the SC state is non-magnetic here in contrast to the AFI state in $\kappa$-(ET) $)_{2} X[17]$. This can be understood theoretically as follows. There exists a strong dimerization as in $\kappa$-(ET) $)_{2} X$, which will result in a Mott insulating state where $\mathrm{S}=1 / 2$ are localized on each dimer, which is inferred from the HF calculations[18]. The spatial arrangement of BETS molecules result in the alternation in the resultant superexchange interactions along the stacking direction together with nonnegligible interchain couplings. The phase diagram of such a spin sys- 
tem in two-dimension has been studied[19], where the ground states are either the AF or the SG state. From the actual parameters deduced from the structure, it is argued that $\lambda$-BETS system is located very close to the boundary between these two states, the latter of which is the candidate here[18].

\section{Summary and Discussion}

The results of recent theoretical studies searching for systematic understanding of the variety of ground states realized in $(\mathrm{TMTCF})_{2} X$ and $(\mathrm{ET})_{2} X$ salts are introduced. Based on the spin dependent Hartree-Fock approximation for the Coulomb interaction together with the consideration of the full anisotropy of the transfer integrals, the key parameters characterizing each family (polytype) are extracted resulting in the coherent understanding of the apparently unrelated types of the ground states. These viewpoints on the organic quarter-filled systems are also applicapable to some transition metal oxides with quarter-filled band, as has been demonstrated in our recent work on $\mathrm{NaV}_{2} \mathrm{O}_{5}[20]$.

The calculations so far, however, have been limited to cases where only one molecular orbital, either LUMO or HOMO, is relevant. There exist other interesting cases where the overlap between LUMO and HOMO appears to be important as in $M$ (dmit) $)_{2}(M=\mathrm{Pd}, \mathrm{Ni})$ compounds or the existence of the mixing between $\pi$-orbital and $d$-orbital plays crucial roles as in $(\mathrm{DCNQI})_{2} \mathrm{Cu}$ and in $\lambda$ (BETS $)_{2} X$ with $X=\mathrm{FeX}_{z} \mathrm{Y}_{4-z}$. The studies on the electronic properties of these systems with multiple orbital degrees of freedom share a common feature with those in the transition metal oxides with orbital degeneracy such as $\mathrm{LaMnO}_{3}$ and $\mathrm{SrRuO}_{4}$ systems, and these will be a challenging problem in the near feature.

\section{Acknowledgement}

The authors are greatful for K. Kanoda, R. Kato, T. Takahashi and many other experimentalists for valuable interactions. HS and HK are supported by the JSPS Research Fellowships for Young Scientists.

\section{References}

[1] H. Kino and H. Fukuyama, J. Phys. Soc. Jpn. 65, 2158 (1996).

[2] D. Jérome, Science 252, 1509 (1991).

[3] H. Seo and H. Fukuyama, J. Phys. Soc. Jpn. 66, 1249 (1997).

[4] T. Nakamura et al, Synth. Metals 70, 1293 (1995).

[5] F. Nad' et al, Eur. Phys. J. B 3, 301 (1998).

[6] K. Hiraki and K. Kanoda, Phys. Rev. Lett. 80, 4737 (1998).

[7] K. Kanoda, Hyperfine interact. 104, 235 (1997).

[8] H. Kino and H. Fukuyama, J. Phys. Soc. Jpn. 64, 1877 (1995); ibid 2726; ibid 4523.

[9] R. McKenzie, Science 278, 820 (1997); Comments Cond. Mat. Phys. 18, 309 (1998).

[10] K. Bender et al, Mol. Cryst. Liq. Cryst. 107, 45 (1984); K. Kajita et al, J. Phys. Soc. Jpn. 61, 23 (1992).

[11] B. Rothamael et al, Phys. Rev. B 34, 704 (1986).

[12] H. Seo and H. Fukuyama, this proceedings; H. Seo, in preparation.

[13] T. Nakamura et al, Synth. Metals, in press; K. Miyagawa and K. Kanoda, Synth. Metals, in press.

[14] H. Mori et al, Phys. Rev. B 57, 12023 (1998).

[15] S. Tajima, private communications.

[16] S. Inagaki and H. Fukuyama, J. Phys. Soc. Jpn. 52, 3620 (1983).

[17] H. Kobayashi et al, Phys. Rev. B 56, 8526 (1997).

[18] H. Seo and H. Fukuyama, J. Phys. Soc. Jpn. 66, 3352 (1997).

[19] N. Katoh and M. Imada, J. Phys. Soc. Jpn. 63, 4529 (1994).

[20] H. Seo and H. Fukuyama, J. Phys. Soc. Jpn. 67, 2602 (1998). 